\title{
TRANSMITTER AND RECEIVER LIGHT FIDELITY TECHNOLOGY WITH PRACTICAL CIRCUIT
}

\author{
Raed S. M. Daraghma \\ Department of Electrical and Electronics Engineering, Palestine Technical University, Palestine \\ R.daraghmeh@ptuk.edu.ps
}

\begin{abstract}
Recently, the radio frequency spectrum is turning into congested, standby process to the wireless communication is needed to be suitable for the exponentially growing wireless passage desire. Visible Light Communication (VLC) arrangements supply a standby to the present criterions of wireless transfer of information. The security and high data rate are the main advantages of this technology compared to wireless technology. The major goal of this paper is to design and implement Light Fight (Li-Fi) that can be worked by using light emitting diode (LED) at the transmitter and photo transmitter at the receiver. If the light is found, we can use it as the transmission channel. Li Fi can supply wireless indoor communication. In this work, we sent data by using Li Fi technique for serval meters.
\end{abstract}

Keyword - Li F, LED, Visible Light.

\section{INTRODUCTION}

In general, utilizing Wi-Fi technology for browsing via the internet has been established on a group of difficulties for examples: the transmit of data almost is slow, low impedance to interface, passive influence on human healthy, construction of this type of technology needs high cost and complex, minimum standard of security and privacy for subscribers.

The most significant daily action is transmitting the data from one place to another; the present wireless network that fasten us to the internet is almost slow when numerous appliances are linked. As the figure of instruments that outlet the internet rises, the constant bandwidth obtainable makes it progressively hard to revel raising data transfer rates and link to a secure network. The problem is that the radio waves have small part of the spectrum obtainable for data carrying. We can solve this problem by using $\mathrm{Li}-\mathrm{Fi}$ technique. It is a transmission of data via illumination through an LED bulb that alters in thickness speeder than the human eye. The Li-Fi technique is faster and inexpensive than Wi-Fi technology.

\section{LITERATURE REVIEW}

The major remarkable evolution in the Li-Fi domain is done in [1], the main goal of this work was to increase the VLC data rate. Two teams from the university of Edinburgh and Oxford are concentrating on analogous data transmission employing arranges of LEDs, where each LED sends a distinct data stream.

In [2], author used mix of green, red and blue LEDs to modify the light frequency encoding distinct data channel. The data rate in this case was reached to 500 Mbyte/second.

In [3], author was suggested that According to the Li-Fi organization, it is possible to obtain more than 10 $\mathrm{Gb} / \mathrm{s}$ of data speed, theoretically which would permit a high-definition film to be downloaded in just 30 seconds.

In [4], author was discussed the features of Li-Fi technique, its suitable for short data transmission by using LED illumination, and travelling at much speed up to $224 \mathrm{~Gb} / \mathrm{s}$.

In [5], author has generated a light bulb that tasks with Li-Fi technology on a tiny measure, he was the primer one who carry out a "real-world test.", he demonstrated that the data transfer speeds of almost $1 \mathrm{~Gb} / \mathrm{s}$ or just almost 100 times rapider than the normal Wi-Fi data speed.

In [6], author was discussed the principle work of the Li-Fi Technique, data is fed into an LED light bulb, it then transmits data at high speeds to the photo detector. The receiver into electrical signal then modifies the small variations in the high speed dimming of LED bulbs. The signal is then transformed back into a binary data stream what we would realizes as web, video, and audio implementations that run on internet enables devices.

In [7], author was discussed the main objective of developed Li-Fi, he described the Li-Fi technology by following live video for the primary once at TED global in 2011, and the term" Li-Fi" was coined by him during this talk. 


\section{SYSTEM DESCRIPTION AND HARDWARE}

Three are many elements should be examined while purposing Li-Fi, figure 1 shown the architecture of Li-Fi.

- $\quad$ Light existence

- $\quad$ Line of sight (LOS)

- $\quad$ Using of fluorescent light and LED to achieve better performance

The main circuit components of the transmitter sound be contained these elements:

Phone playing audio

- Audio jack

- Power supply (5V battery or adapter)

- LED (white color)

- Capacitors (22uF)

- Transistors (c1815)

- Resistors (10k, 1k)

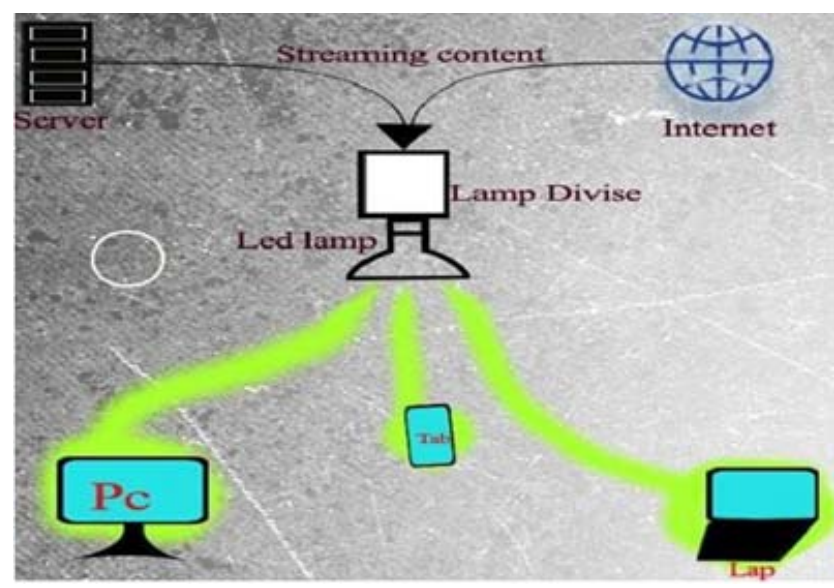

Fig. 1. Architecture of Li-Fi

Figure 2. Shown the block diagram of the transmitter circuit for Li-Fi

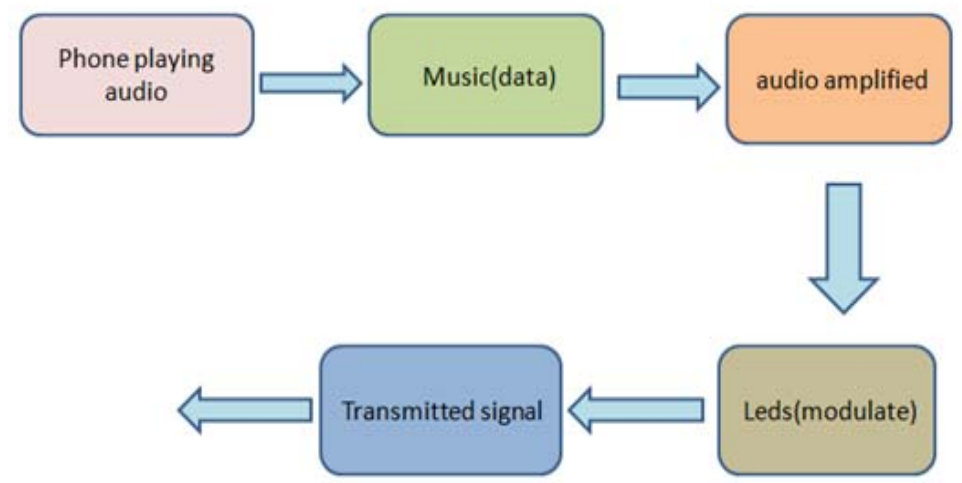

Fig. 2 block diagram of transmitter sound by Li-Fi

Figure 3. Shown the schematic diagram for the trasnmitter circuit, Figure 4. Shown the practice trasnitter circuit. 


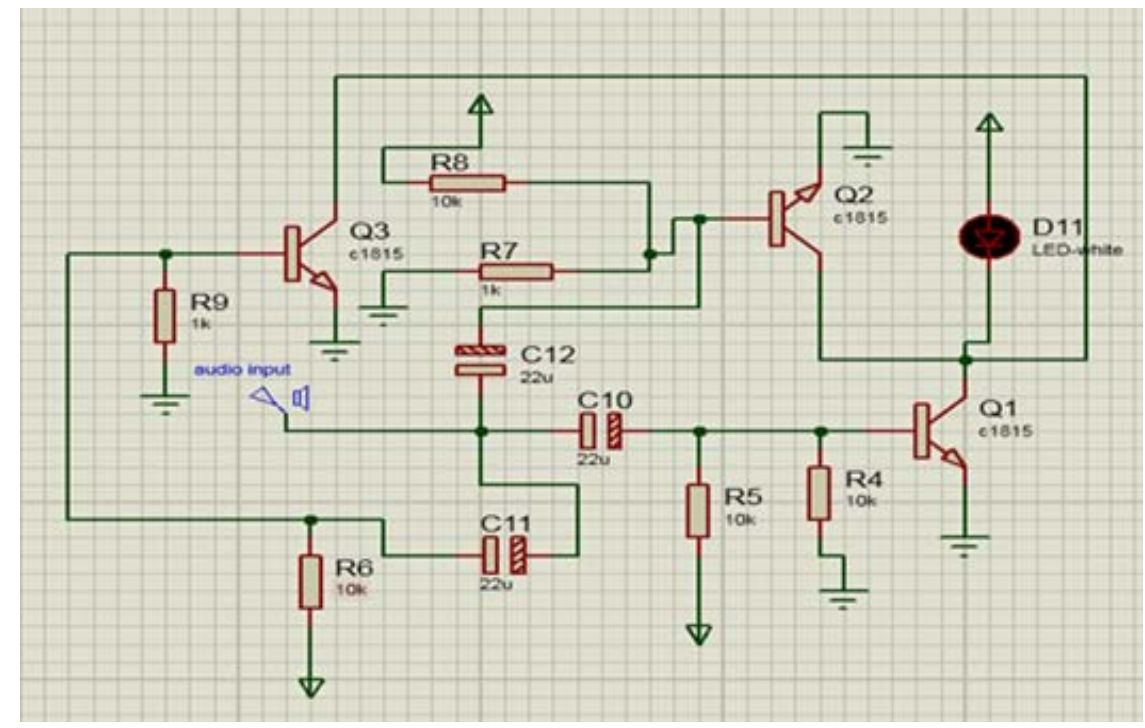

Fig 3. The schematic diagram for the transmitter circuit

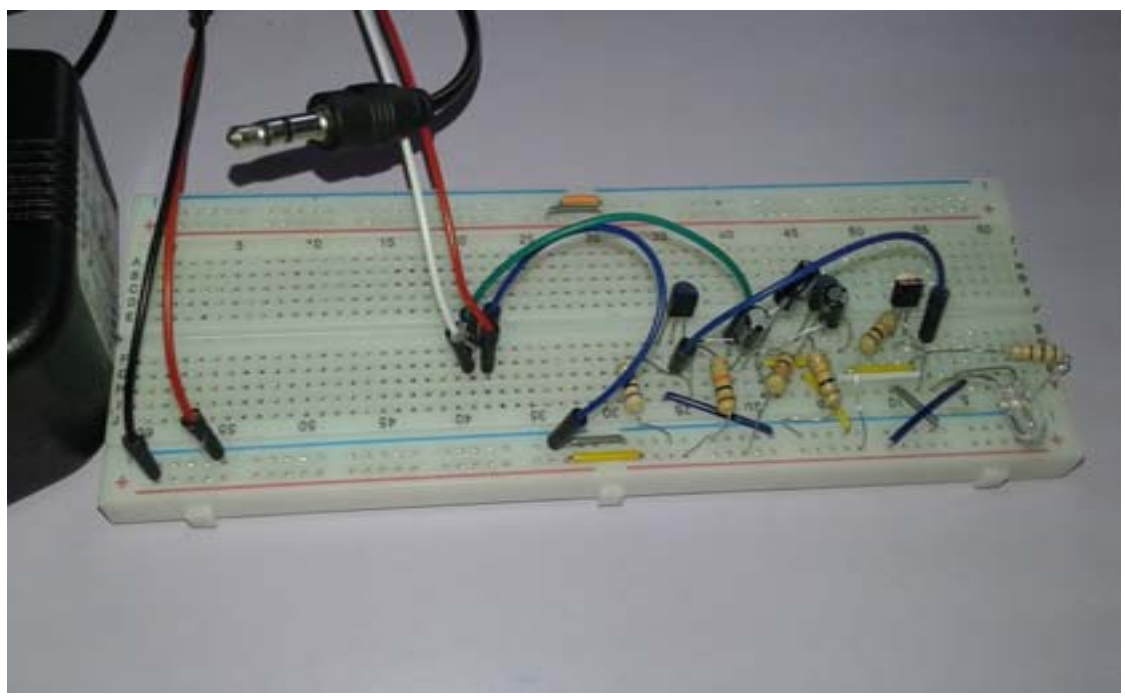

Fig. 4. Practice transmitter Circuit

Figure 5. Shown the main block diagram for the circuit

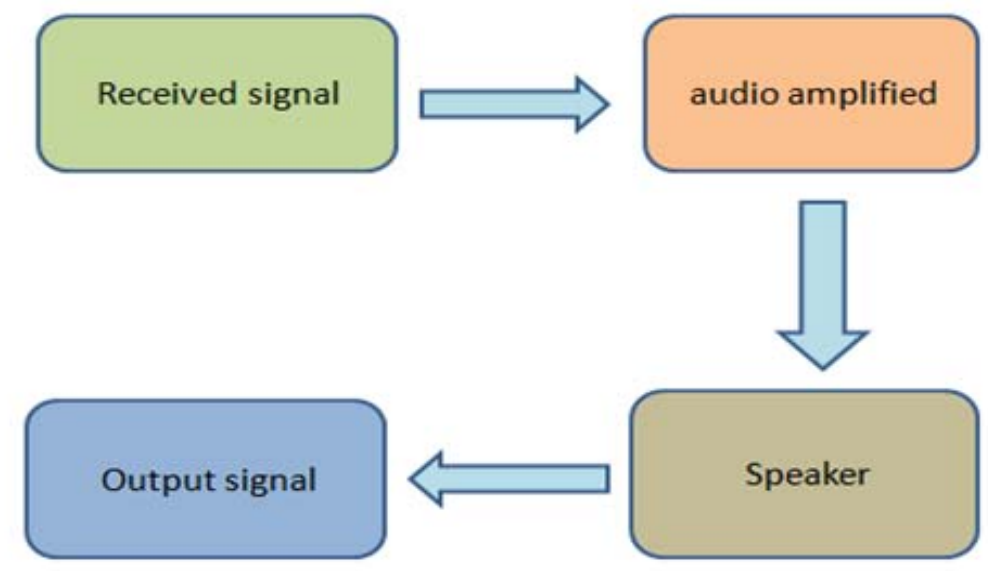

Fig. 5. block diagram of receiver sound by Li-Fi. 
The Table I. Show comparison between LI-FI \& WI-FI:

TABLE I. SHOW COMPARSION BETWEEN LI-FI \& WI-FI:

\begin{tabular}{|c|c|c|}
\hline Characteristic & $\mathrm{Li}-\mathrm{Fi}$ & $\mathrm{Wi}-\mathrm{Fi}$ \\
\hline Speed of data Transfer & $\begin{array}{l}\text { Data Transfer speed } \\
\text { (>1Gbps) }\end{array}$ & $\begin{array}{l}\text { Data Transfer speed } \\
\text { (150Mbos) }\end{array}$ \\
\hline Frequency & No Frequency for Light & $5 \mathrm{GHz}$ \\
\hline Standard & IEEE 802.15 & IEEE 802.11 \\
\hline Range & Base on LED Light & 100 meters \\
\hline Data Transfer rate & $>1$ Gbps & 800Kbps - 11Mbps \\
\hline Power consumption & Low & Medium \\
\hline Network topology & Point to point & Point to point \\
\hline Primary Application & $\begin{array}{c}\text { Wireless local area } \\
\text { networking }\end{array}$ & $\begin{array}{l}\text { Wireless local area } \\
\text { networking Cost Low } \\
\text { Medium high }\end{array}$ \\
\hline Security & High & Medium \\
\hline
\end{tabular}

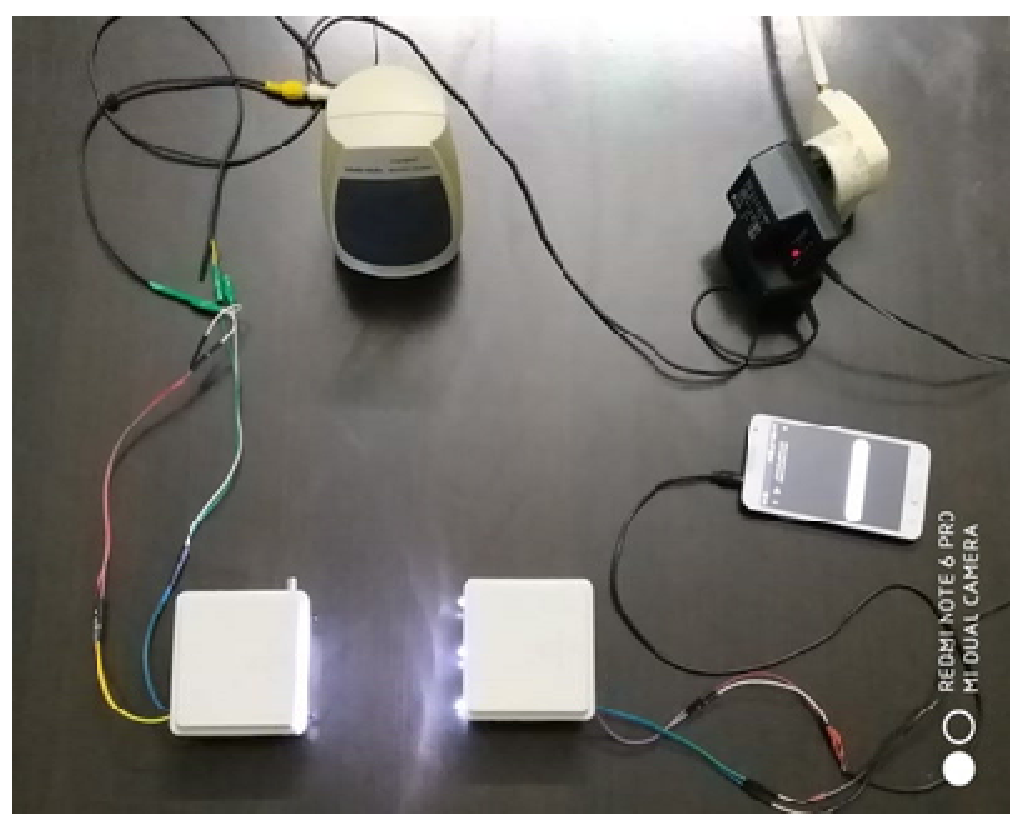

Fig. 6. Final Li-Fi circuit project. 
Table II. Cost of project.

\begin{tabular}{|c|c|c|c|}
\hline Item & Unit price (NIS) & No. of pieces & Total price $\$$ \\
\hline audio jack & 2 & 1 & 0.5 \\
\hline Power supply (9V battery or adapter) & 3 & 1 & 0.8 \\
\hline Breadboard & 13 & 2 & 7 \\
\hline Jumper cable & .33 & 1 & 0.1 \\
\hline LED (white color) & .33 & 1 & 0.1 \\
\hline capacitors (22uF) & 1 & 1 & 0.3 \\
\hline transistors (c1815) & 1 & 1 & 0.3 \\
\hline Resistors (10k) & .33 & 3 & 0.3 \\
\hline photo diode & 5 & 1 & 1.2 \\
\hline Speaker & 5 & 1 & 1.4 \\
\hline potentiometer (10k) & 3 & 1 & 0.8 \\
\hline capacitors $(0.1 \mu \mathrm{F})$ & 1 & 2 & 0.5 \\
\hline capacitors $(10 \mu \mathrm{F})$ & 1 & 1 & 0.3 \\
\hline capacitors $(100 \mu \mathrm{F})$ & 2 & 1 & 0.5 \\
\hline capacitors $(1000 \mu \mathrm{F})$ & 2 & 1 & 0.5 \\
\hline capacitors $(0.033 \mu \mathrm{F})$ & 1 & 1 & 0.3 \\
\hline capacitors(470pF) & 1 & 1 & 0.3 \\
\hline lm386 audio amplifier & 3 & 1 & 0.3 \\
\hline \multicolumn{4}{|c|}{ Total Price $=15.5 \$$} \\
\hline
\end{tabular}

\section{CONCLUSION}

Li-Fi technology is established on LEDs or other light exporter for the transmitting of data. This exporter may be invisible, ultraviolet or the visible part of the spectrum. We can conclude that the Li-Fi technology has many advantages: high-speed data could be hundred times of radio waves; LED or other light exporter consumes less energy, high efficient. Light exporters are existing everywhere; this technology cannot be interrupted or misused. Connecting a large number of subscriber is very easy with high-speed capability. In the contrary, this technology has major limitations such as: if there is obstacle between the transmitter and receiver, we cannot transfer data, i.e. the transmitter antenna and receiver antenna should be in line. It requires Line of Sight.

In our project, we achieve the project objectives that transferring audio data in an easy, simple and secure way. We designed and implemented a wireless communication device, which transmits audio message wirelessly using LED (Li-Fi). The project contains two sections: 1- Transmitter Section 2- Receiver Section. The transmitter section modulates the incoming message audio signal and transmits towards the receiver in the form of visible light using LEDs. The receiver section interprets the incoming light, which is detected using a photo diode, and converts to the audible sound signal with the help of speaker. If Li-Fi technology will be use practically in the future, Wi-Fi hotspot will be used as transmitter to send wireless information for every bulb, as a result, the future will be safer, cleaner, greener and safer.

\section{AKNOWLEDEGEMENT}

We would say to thank for those supports our research. We sincerely thank the anonymous reviewers for their constructive comments and suggestions. I never forget, my deep thank for my university: Palestine Technical University Kadoorei. 


\section{REFERNCES}

[1] Vaishali Jadhav, "A Study on Li-Fi - Light Fidelity Technology “, International Journal of Scientific \& Engineering Research, Volume 5, Issue 6, June-2014.

[2] V. M. Deshmukh, B. Meshram, "Serial Data Transmission using Visible Light Communication “, International Journal on Future Revolution in Computer Science \& Communication Engineering, Volume 4, Issue 3, March 2018.

[3] N. Navyatha, T. Prathyusha, M. Mounika, "Li-Fi (Light fidelity)-LED Based Alternative ", International Journal of Scientific \& Engineering Research, Volume 4, Issue 5, May-2013.

[4] M. Bhalerao, S. Sonavane, V. Kumar, "A Survey of Wireless Communication Using Visible Light “, International Journal of Advances in Engineering \& Technology, Vol. 5, Issue. 2, 2013

[5] V. Navgire, D. Kathole, R. Telgad, "A Review: Li-Fi Technology (The Future of the Internet) “, IOSR Journal of Computer Engineering (IOSR-JCE), e-ISSN: 2278-0661, p- ISSN: 2278-8727.

[6] R Jain, P. vikale, P. Kadam," "Wireless Data Communication Using Li-Fi Technology “, IOSR Journal of Electrical and Electronics Engineering (IOSR-JEEE), Volume 11, Issue 2 Ver. II (Mar. - Apr. 2016), PP 65-68.

[7] R. Sharma, R. Sanganal, "Li-Fi Technology Transmission of Data through Light “, IJCTA, Volume 3, Issue 2, March- April, 2015, pp. 150-154.

\section{AUTHOR}

RAED S. M. DARAGHMA was born in 1977 in Palestine, he received his master degree from Jordan science and technology in electrical and communication engineering Jordan in 2010, and he got his P.H.D degree from Anadolu University, turkey in 2016. He has published number of papers and journals. He has engaged in educational work many years manly teaches digital communication, mobile, digital communication networks at Palestine technical university P.T.U. His mainly research areas include wireless sensor networks, signal processing and MIMO radar. 